\title{
Magnetic-field-induced enhancement of ion beam energy in a magnetically expanding plasma using permanent magnets
}

\author{
Kazunori Takahashi, Yutaka Shida, and Tamiya Fujiwara \\ Department of Electrical and Electronic Engineering, Iwate University, Morioka \\ 020-8551, Japan \\ E-mail: kazunori@iwate-u.ac.jp
}

\begin{abstract}
Ion energy distribution functions (IEDFs) near a source exit of a magnetically expanding argon plasma using only permanent magnets (PMs) are measured by a combination of a retarding field energy analyzer and a pulsed probe method for three types of magnetic-field configurations and various operating gas pressures, where the magnetic-field strength in the source tube is increased up to 270 Gauss by adding the number of the PM layers. The $13.56 \mathrm{MHz}$ rf power for plasma production is maintained at $250 \mathrm{~W}$. The IEDFs show the existence of an accelerated group of ions. It is found that the energy of the accelerated ions increases when the magnetic-field strength is increased, but saturates at seven times the electron temperature at argon pressures of 0.6-1.6 mTorr. Our results show that the maximum velocity of the accelerated ions is found to be $14 \mathrm{~km} / \mathrm{sec}$ with a Mach number of 3.8 .
\end{abstract}




\section{Introduction}

Expanding plasmas containing electric double layers (DLs) have been vigorous research subjects in connection with particle acceleration in space and laboratory plasmas, and electric propulsion devices $[1,2,3,4,5,6]$. In recent laboratory experiments, supersonic ion beams accelerated by the DLs have been detected in magnetically expanding rf plasmas using retarding field energy analyzers (RFEAs) [7, 8] and laser induced fluorescence methods $[9,10,11]$. It is reported that this type of DLs is maintained by only the single plasma source, where the upstream energetic electrons overcoming the potential drop of the DL neutralize the ion beam accelerated by the DL $[12,13,14]$. The electrodeless ion acceleration mechanism is proposed to be utilized for development of a new type of electrodeless long-lifetime propulsion device $[5,15]$. The previously reported ion beam energies or DL strengths were typically five times the electron temperature (5 $T_{e}$ ) measured at the downstream side of the DL or in the range of 3-7 $T_{e}[4,8,16]$. Since the thrust force of the propulsion device is related to the ion beam energy, it is important to study the detailed behavior of the ion beam energy for various conditions, e.g., the gas pressure, magnetic-field strength, rf power. Recently, the detailed role of the magnetic-field strength has been experimentally investigated by changing the solenoid currents; the ion beam energy and the DL strength are observed to increase with an increase in the magnetic-field strength in the source tube and saturate at about $5 T_{e}$ for field strength above several tens of Gauss [17].

In studies of ion acceleration in expanding double-layer plasmas, the solenoids are used to provide the diverging magnetic field. Recently, authors have reported the generation of the supersonic ion beam in a solenoid-free plasma expanded by permanent magnets (PMs) $[18,19]$. The use of the PMs would lead to a reduction of electric power consumption and of the weight of the propulsion device. The constant field region in the source tube and the diverging field region near the source exit can be created by double concentric arrays of PMs; the magnetic-field strength in the source tube was about 100 Gauss. A rapid potential drop over a thickness of 100-200 Debye length is formed near the source exit and the accelerated ion beam with energy corresponding to the potential drop is detected. In order to apply this source to the propulsion device, the detailed characteristics of the accelerated ion beam need to be investigated for various operating conditions.

In the present paper, the ion energy distribution functions (IEDFs) near the source exit of the magnetically expanding plasma using PMs are measured for three types of magnetic field strengths under various operating gas pressures, where the magnetic-field strength can be increased by adding the number of the PM layers. The enhancement of ion beam energy for strong magnetic fields is observed in the present experiments, but the energy appears to saturate at about $7 T_{e}$ for stronger magnetic fields above 200 Gauss. The maximum observed ion velocity is found to be about $14 \mathrm{~km} / \mathrm{sec}$ with a Mach number of 3.8. 


\section{Experimental setup}

\subsection{Machine configuration}

A schematic diagram of the experimental setup is shown in Fig. 1. A cylindrical glass tube of about $20 \mathrm{~cm}$ in length and $6.5 \mathrm{~cm}$ in inner diameter (source tube) is attached contiguously to a 26-cm-diameter and 30-cm-long grounded stainless steel vacuum chamber (diffusion chamber). The chamber is evacuated to a base pressure of $2 \times 10^{-6}$ Torr by a diffusion/rotary pumping system, and the argon gas is introduced from the upstream flange of the source tube through a mass flow controller. The argon gas pressure in the vacuum chamber is maintained in the range of 0.6-1.6 mTorr, where the gas pressure is measured by the ionization gauge connected to a sideport of the diffusion chamber. A steady-state argon plasma is excited by a 7.5-cm-diameter twoturn loop antenna located at $z=-9 \mathrm{~cm}$ and powered from a rf power supply of frequency $13.56 \mathrm{MHz}$ and power $250 \mathrm{~W}$, where $z=0$ is defined as the interface between the source tube and the diffusion chamber. Surrounding the source tube and the rf antenna, two concentric arrays of PMs are arranged for generation of diverging magnetic-field configurations. The detailed magnetic-field configurations are described in Sec. 2.2. An insulator plate located near the upstream flange terminates the plasma cavity at the upstream side. Under these conditions, we have already reported that the rapid potential drop of the DL is spontaneously generated near the source exit $(z \sim-2-1 \mathrm{~cm})$ and the ion beam with an energy corresponding to the potential drop of the DL is detected in the downstream diffusion chamber [18]. In order to measure the IEDFs downstream of the DL, the RFEA is located at $z=3 \mathrm{~cm}$. The RFEA consists of an electron reflector mesh biased at $-60 \mathrm{~V}$, an ion collector electrode, and a 3-mm-diameter entrance orifice facing the source tube. Then, the IEDF is proportional to the first derivative of the collector current $I_{c}$ - collector voltage $V_{c}$ curve [20], which are obtained through the pulsed probe technique [21] described in Sec. 2.3.

\subsection{Magnetic-field configurations}

In general, the magnetic fields produced by the PMs are strongly nonuniform with reverse-fields, i.e., cusps. According to experiments performed by Shamrai et al., the cusp fields prevent the plasma diffusion, the formation of the DL, and the generation of the subsequent ion beam in the expanding plasmas [22]. Thus, it is required that the magnetic-field configuration has a constant-field region in the source tube and a diverging-field region without any cusp fields. In order to provide the above-mentioned fields, double concentric arrays of PMs as shown in Fig. 2(a) are set around the source tube. In the present experiment, all of the magnets are Neodymium Iron Boron $(\mathrm{NdFeB})$ magnets of $10 \mathrm{~cm}$ in length, $1.5 \mathrm{~cm}$ in width, and $0.5 \mathrm{~cm}$ in thickness. All of the magnets have inward magnetization in the radial direction, i.e., in the direction of thickness. The top and the bottom of Fig. 2(b) show the side view of the sketches of the magnetic-field lines produced by the single array and the double arrays of PMs, respectively. As shown 
at the top of Fig. 2(b), the single array of PMs can produce the diverging magnetic fields, but the cusp fields also exist at the axial center of the array. Between the cusp and the diverging fields, there is no constant field region for the case of the single array. On the other hand, the double arrays can create the constant field region in addition to the diverging fields as shown at the bottom of Fig. 2(b). By employing this double magnet-arrays around the source tube, the expanding magnetic fields can be applied to the machine.

In the present experiment, three types of arrangement of the magnets as shown in Fig. 3(a) are used, which are labeled as configs. (I), (II), and (III), respectively. The numbers $\left(N_{\text {inner }}, N_{\text {outer }}\right)$ of the PMs used for the inner and outer arrays are $(8,8)$ for config. (I), $(24,24)$ for config. (II), and $(24,32)$ for config. (III). The calculated axial profiles of the magnetic-field strength $B_{z}$ in the axial direction are plotted in Fig. 3(b) as a solid line, a dotted line, and a dotted-dashed line for configs. (I), (II), and (III), respectively. The magnetic-field strengths in the source tube for configs. (I), (II), and (III) are found to be fairly uniform and are about 100, 200, and 270 Gauss, respectively. Furthermore, it is clear the diverging magnetic fields can be provided near the source exit without any cusp fields.

\subsection{Pulsed probe technique for IEDF measurement}

An electronic technique for the measurement of the IEDF, i.e., the first derivative of $I_{c}-V_{c}$ curve, is described. When the collector voltage $V_{c}$ is linearly swept $\left(d V_{c} / d t=\right.$ constant $), d I_{c} / d t$ is proportional to $d I_{c} / d V_{c}$, where $t$ is time. Therefore, the IEDF can be directly obtained by the measurement of the first derivative of $I_{c}$ - $t$ characteristic when $V_{c}$ is linearly swept. Figure 4(a) shows the measurement circuit of the IEDF. Outside the plasma, the current signal is passed through a resistor and then is connected to a bipolar voltage source that is swept for about $25 \mathrm{msec}$ from 110 $\mathrm{V}$ to $-40 \mathrm{~V}$. The voltage signal from the resistor, i.e., the current signal, is fed through an isolation amplifier into an active analog circuit called differentiator; the detailed circuit diagram is shown in Fig. 4(b). The voltage gain $G_{v}\left(\equiv v_{\text {out }} / v_{\text {in }}\right)$ of the circuit in Fig. 4(b) can be derived as

$$
\begin{aligned}
G_{v} & =-\frac{R_{2}}{R_{1}} \frac{1}{\left(1+1 / j \omega C_{1} R_{1}\right)\left(1+j \omega C_{2} R_{2}\right)}, \\
& =-\frac{R_{2}}{R_{1}} \frac{1}{(1+1 / j \omega T)(1+j \omega T)},
\end{aligned}
$$

where $R_{1}, R_{2}, C_{1}, C_{2}$ are the circuit parameters in Fig. 4(b), and $T$ is defined as $T \equiv R_{1} C_{1}=R_{2} C_{2}$ for simplification. In this experiment, the circuit parameters are set as $R_{1}=1 \mathrm{k} \Omega, C_{1}=200 \mathrm{nF}, R_{2}=100 \mathrm{k} \Omega$, and $C_{2}=2 \mathrm{nF}$, respectively. For low $(\omega T \ll 1)$ and high $(\omega T \gg 1)$ frequency conditions, the gain can be simplified as $G_{v}=-j \omega C_{1} R_{2}$ and $G_{v}=-1 /\left(j \omega C_{2} R_{1}\right)$, respectively. Hence, the active circuit acts as differentiator for the current signal during slow voltage sweep and as as a filter for electric noises above a few $\mathrm{kHz}$. The differentiated signal is digitized by a digital storage oscilloscope, 
and passed into a LABVIEW program for conversion into ASCII data and display on a computer.

Figure 5(a) shows the representative results of the collector bias voltage $V_{c}$ (dotteddashed line), collector current $I_{c}$ (dotted line), and the first derivative $d I_{c} / d t$ (solid line) as a function of time $t$, where the data is averaged over many shots. From the data set of the measured $V_{c}$ and $d I_{c} / d t$, we can obtain the IEDF shown in Fig. 5(b). The IEDF in Fig. 5(b) is found to have the bulk ions at $V_{c} \sim 20 \mathrm{~V}$ and the accelerated group of ions at $V_{c} \sim 50 \mathrm{~V} ; V_{c}$ yielding these peaks in IEDF correspond to the local plasma potential $\phi_{p}$ and the beam potential $\phi_{\text {beam }}$. Here, we note that the beam potential is different from the beam energy. Since $V_{c}$ is the voltage from the electrical ground, zero energy corresponds to the local plasma potential, and the ion beam energy $\varepsilon_{\text {beam }}$ can be estimated as $\varepsilon_{\text {beam }} \equiv \phi_{\text {beam }}-\phi_{p}$.

\section{Experimental results}

Figures 6(a), 6(b), and 6(c) show the normalized IEDFs measured at $z=3 \mathrm{~cm}$ for configs. (I), (II), and (III), respectively, where the argon gas pressure and the rf power are maintained at $1 \mathrm{~m}$ Torr and $250 \mathrm{~W}$. It has previously been reported by authors that the energy of the accelerated group of ions is in good agreement with the potential difference between the upstream and downstream plasmas, where the potential drop of the DL is spontaneously generated near the source exit [18]. The local plasma potential $\phi_{p}$ and the beam potential $\phi_{\text {beam }}$ are indicated by solid and dashed arrows in Fig. 6 . The ion beam energies $\varepsilon_{\text {beam }}=\phi_{\text {beam }}-\phi_{p}$ for configs. (II) and (III), i.e., for stronger magnetic-field configurations, are found to be higher than that for config. (I).

The ion beam energies $\varepsilon_{\text {beam }}$ as a function of the argon gas pressure $P_{A r}$ for configs. (I), (II), and (III) are plotted in Fig. 7(a) as closed squares, crosses, and open circles, respectively. For all magnetic-field configurations, it is found that the ion beam energy increases with a decrease in the gas pressure $P_{A r}$; this behavior is very similar to the previously reported results in the magnetically expanding plasmas using electromagnets, and the diffusion-controlled model [23]. Furthermore, the results in Fig. 7(a) show that the ion beam energy can be enhanced by stronger magneticfield configurations such as configs. (II) and (III). Since there is no change of the ion beam energy between the results for configs. (II) and (III), the beam energy appears to saturate for the magnetic-field strength above about 200 Gauss.

The velocity $v_{\text {beam }}$ of the beam ion is evaluated using [7]

$$
v_{\text {beam }}=\sqrt{\frac{2 e \varepsilon_{\text {beam }}}{M_{i}}},
$$

where $M_{i}$ is the ion mass. The beam velocities $v_{\text {beam }}$ obtained from the results in Fig. 7(a) are presented in Fig. 7(b); the maximum of the beam velocity is about 14 $\mathrm{km} / \mathrm{sec}$ for $P_{A r}=0.6$ mTorr under configs. (II) and (III).

The potential drop $\phi_{D L}$ of the DL and the ion beam velocity are evaluated by the strength $\phi_{D L} / T_{e}$ of the DL and the Mach number, where $\phi_{D L}$ is observed to be equal 
to the energy $\varepsilon_{\text {beam }}$ of the ion beam. In order to discuss these values, the electron temperature $T_{e}$ at the downstream side of the DL is measured at $z=10 \mathrm{~cm}$ by a singletipped Langmuir probe, where $T_{e}$ is obtained from the IV curve under the assumption of the Maxwellian electron energy distribution function (EEDF). Figure 8 shows the measured electron temperatures $T_{e}$ versus the argon gas pressure $P_{A r}$. The electron temperature $T_{e}$ is found to be independent of the magnetic-field configuration, and decreases from about $6 \mathrm{eV}$ to $3 \mathrm{eV}$ when the gas pressure $P_{A r}$ is increased from 0.6 mTorr to 1.7 mTorr.

From the results shown in Figs. 7 and $8, \varepsilon_{\text {beam }} / T_{e}$ and a Mach number are obtained as shown in Figs. 9(a) and 9(b), respectively. Here the Mach number is derived as $v_{\text {beam }} / C_{s}$, where $C_{s}$ is the ion sound speed and defined as $C_{s}=\sqrt{k_{B} T_{e} / M_{i}}$. In Fig. 9(a), $\varepsilon_{\text {beam }} / T_{e}$ for various gas pressures is found to increase when the magnetic-field strength is increased to 200 and 270 Gauss, i.e., configs. (II) and (III). However, $\varepsilon_{\text {beam }} / T_{e}$ corresponding to the DL strength saturates at about $7 T_{e}$. In addition, $\varepsilon_{\text {beam }} / T_{e}$ is independent of the gas pressure for configs. (II) and (III), while it is reduced from 5 to 3 for config. (I) when the pressure is increased from 0.6 mTorr to 1.6 mTorr. A possible reason for the reduction of $\varepsilon_{\text {beam }} / T_{e}$ for config. (I) is that the effects of the magneticfield would be lost in the plasma expansion for high-pressure and low-magnetic-field condition; the plasma flow becomes more diffusive and the strength of the DL triggered by the expanding magnetic field is reduced.

In a previously reported one-dimensional model of the DL in magnetically expanding plasmas, based on the sheath theory with the assumption of a Maxwellian EEDF, it has been predicted that the maximum strength of the DL is about $5 T_{e}$ for argon plasmas [24]. Then the flux of the electrons passing through the DL is equal to the flux of the accelerated ions. According to this model, there would not be sufficient electrons overcoming the potential drop of the DL and neutralizing the accelerated ion beam if the potential drop exceeds $5 T_{e}$. In the present experiments using the PMs, the strength of the DL is about $7 T_{e}$, being higher than the predicted one in Ref. [24]. Moreover, the observed ion beam energy appears to be higher than the previously reported one in the magnetically expanding plasmas using electromagnets [17]. Another experiment performed in WONBAT [8] has shown the DL with the strength of $7 T_{e}$ only for the gas pressure below 0.1 mTorr. The present experiment shows the DL strength of $7 T_{e}$ for various gas pressures under the strong magnetic-field configurations. Although the physical mechanisms for the DL strength in the present experiment is unclear now, it is possible that the complicated magnetic-field configuration provided by the PMs could affect the potential drop of the DL and the higher-energy ion acceleration. In the present magnetic-field configuration, the magnetic-field strength is azimuthally nonuniform; hence the electrons are trapped by the magnetic fields terminated by the glass wall and heated by the rf electric fields for the plasma production. Therefore, it is possible that the energetic tail electrons is more likely in the source tube, which can overcome the potential drop of the DL and flow into the downstream plasma. Although the EEDF is treated as Maxwellian for obtaining $T_{e}$ in the present experiments, the 
detailed measurement of the EEDF is required in the future. In the previously reported DL plasmas using electromagnets [7], the Mach number of the accelerated ions is about 2.1 , which is evaluated by the electron temperature downstream of the DL. In the present machine with the PMs, on the other hand, the maximum of the Mach number of the accelerated ion beam is found to be about 3.8. Hence, the expanding magnetic-field configuration provided by the PM arrays would be more effective for the generation of the high-speed ion beam. In addition to the above-mentioned consideration, the difference in the source diameter needs to be taken into account. The effect of the source diameter also has to be investigated hereafter.

\section{Conclusion}

The ion energy distribution functions (IEDFs) downstream of the double layers (DLs) are investigated in a magnetically expanding solenoid-free argon plasma using permanent magnets (PMs) under three types of magnetic-field configurations of about 100, 200, and 270 Gauss in the source tube. The IEDFs can be obtained by the combination of the retarding field energy analyzer (RFEA) and the pulsed probe technique. The IEDFs near the source exit clearly show the existence of the accelerated group of ions. The energy of the accelerated ions, which indicates the potential drop of the DL, is observed to increase with a decrease in the argon gas pressure. The energy can also be increased by increasing the upstream magnetic-field strength from previously reported 100 Gauss to above 200 Gauss in the present experiments, although the energy saturates at seven times the electron temperature. The maximum velocity of the accelerated ions is about $14 \mathrm{~km} / \mathrm{sec}$ and 3.8 in Mach number.

\section{Acknowledgements}

This work was supported in part by Grant-in-Aid for Young Scientists (B, 20740317) from the Ministry of Education, Culture, Sports, Science and Technology, Japan, by the Yazaki Memorial Foundation for Science and Technology, and by the Foundation for the Promotion of Ion Engineering. 


\section{References}

[1] Hairapetian G and Stenzel R L 1988 Phys. Rev. Lett. 611607

[2] Ergun R E, Su Y -J, Andersson L, Carlson C W, McFadden J P, Mozer F S, Newman D L, Goldman M V, and Strangeway R J 2001 Phys. Rev. Lett. 87045003

[3] Boswell R W, Marsch E, and Charles C 2006 Astrophys. J. 640 L199

[4] Charles C 2007 Plasma Sources Sci. Technol. 16 R1 and references therein

[5] Charles C 2009 J. Phys. D: Appl. Phys. 42163001 and references therein

[6] Ergun R E, Andersson L, Tao J, Angelopoulos V, Bonnell J, McFadden J P, Larson D E, Eriksson S, Johansson T, Cully C M, Newman D N, Goldman M V, Roux A, LeContel O, Glassmeier K -H, and Baumjohann W 2009 Phys. Rev. Lett. 102155002

[7] Charles C and Boswell R W 2004 Phys. Plasmas 111706

[8] Sutherland O, Charles C, Plihon N, and Boswell R W 2005 Phys. Rev. Lett. 95205002

[9] Cohen S A, Siefert N S, Stange S, Boivin R F, Scime E E, and Levinton F M 2003 Phys. Plasmas 102593

[10] Sun X, Keesee A M, Biloiu C, Scime E E, Meige A, Charles C, and Boswell R W 2005 Phys. Rev. Lett. 95025004

[11] Thakur S C, Harvey Z, Biloiu I A, Hansen A, Hardin R A, Przybysz W S, and Scime E E 2009 Phys. Rev. Lett. 102035004

[12] Meige A and Boswell R W 2006 Phys. Plasmas 13092104

[13] Takahashi K, Charles C, Boswell R W, Kaneko T, and Hatakeyama R 2007 Phys. Plasmas 14 114503

[14] Takahashi K, Charles C, Boswell R W, and Hatakeyama R 2008 Phys. Plasmas 15074505

[15] West M D, Charles C, and Boswell R W 2008 J. Propul. Power 24134

[16] Charles C and Boswell R W 2003 Appl. Phys. Lett. 821356

[17] Charles C and Boswell R W 2007 Appl. Phys. Lett. 91201505

[18] Takahashi K, Oguni K, Yamada H, and Fujiwara T 2008 Phys. Plasmas 15084501

[19] Takahashi K and Fujiwara T 2009 Appl. Phys. Lett. 94061502

[20] Takahashi K, Kaneko T, and Hatakeyama R 2006 Plasma Sources Sci. Technol. 15495

[21] Schoenberg K F 1978 Rev. Sci. Instrum. 491377

[22] Shamrai K P, Virko Y V, Virko V F, and Yakimenko A I, Proceedings of the 42nd AIAA/ASME/SAE/ASEE Joint Propulsion Conference and Exhibit, California 2006, (the American Institute of Aeronautics and Astronautics, 2006), p. 4845.

[23] Lieberman M A, Charles C, and Boswell R W 2006 J. Phys. D: Appl. Phys. 393294

[24] Chen F F 2006 Phys. Plasmas 13034502 


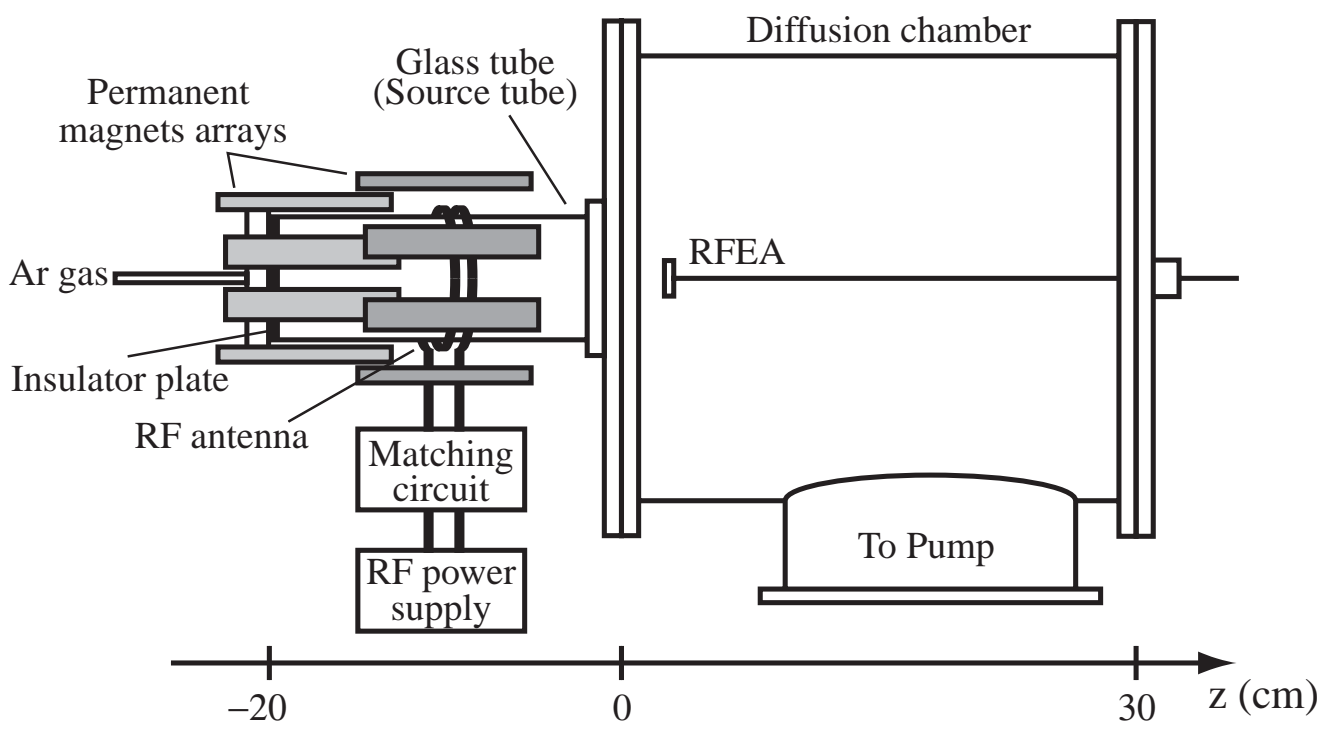

Figure 1. Schematic of the experimental setup. 
(a)

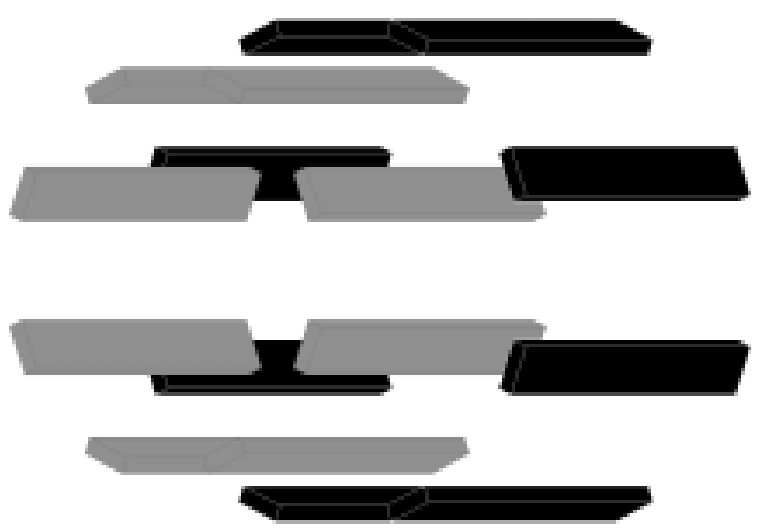

(b)
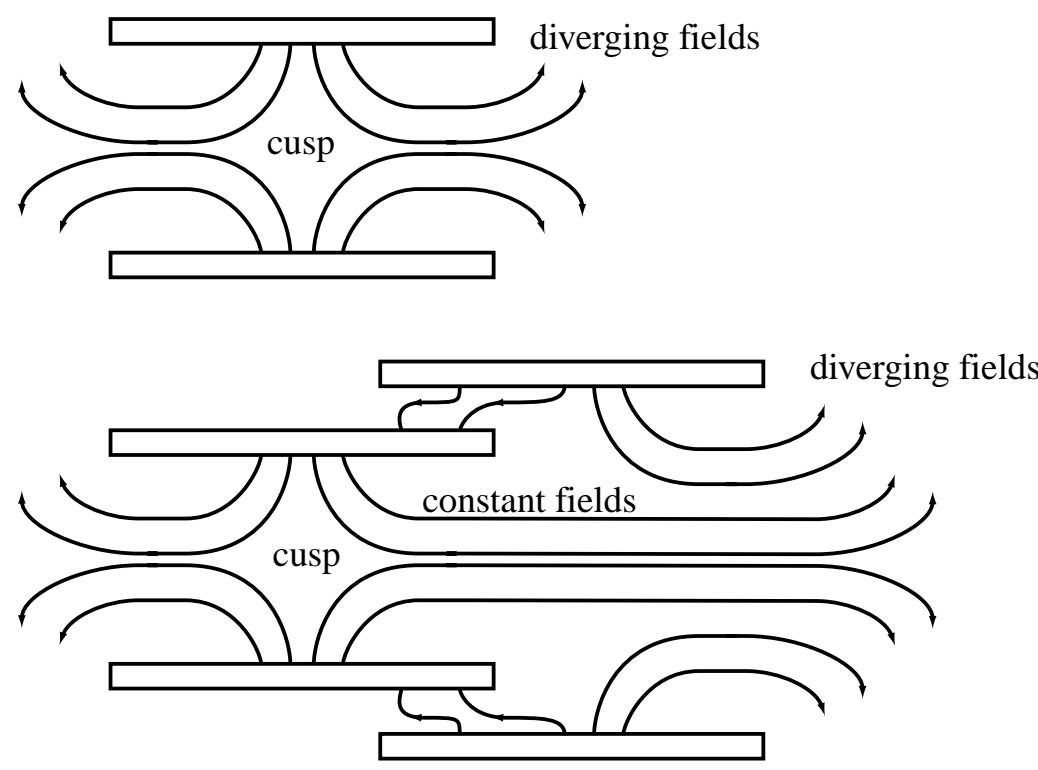

Figure 2. (a) Schematic view of the double concentric arrays of PMs. (b) Images of the magnetic-field lines created by the single magnet-array (top) and the double magnet-arrays (bottom). 
(a)

config. (I)

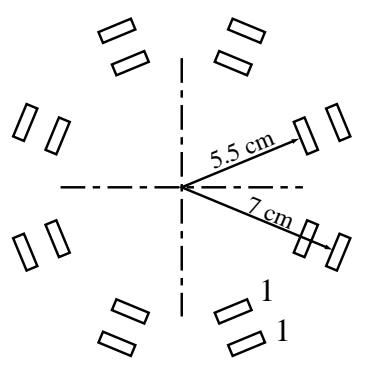

config. (II)

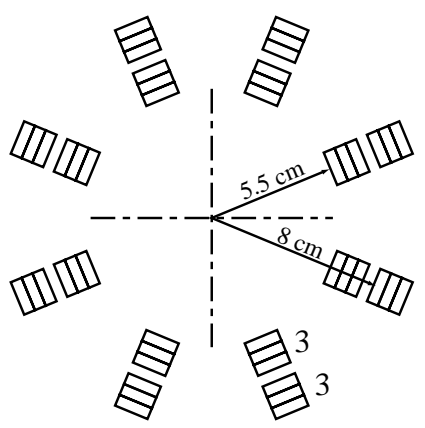

config. (III)

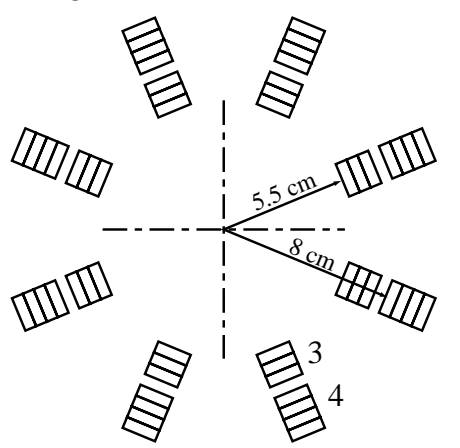

(b)

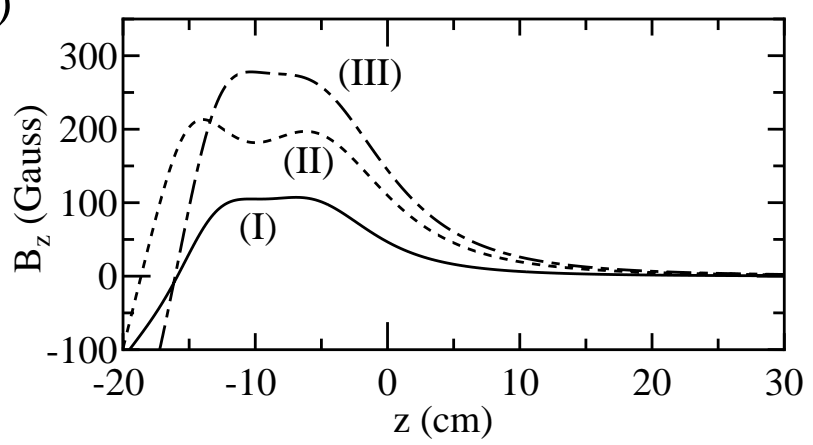

Figure 3. (a) Axial view of the three types of the PMs arrays, which are labeled as configs. (I), (II), and (III). (b) The calculated magnetic-field configurations for configs. (I) (solid line), (II) (dotted line), and (III) (dotted-dashed line). 
(a)

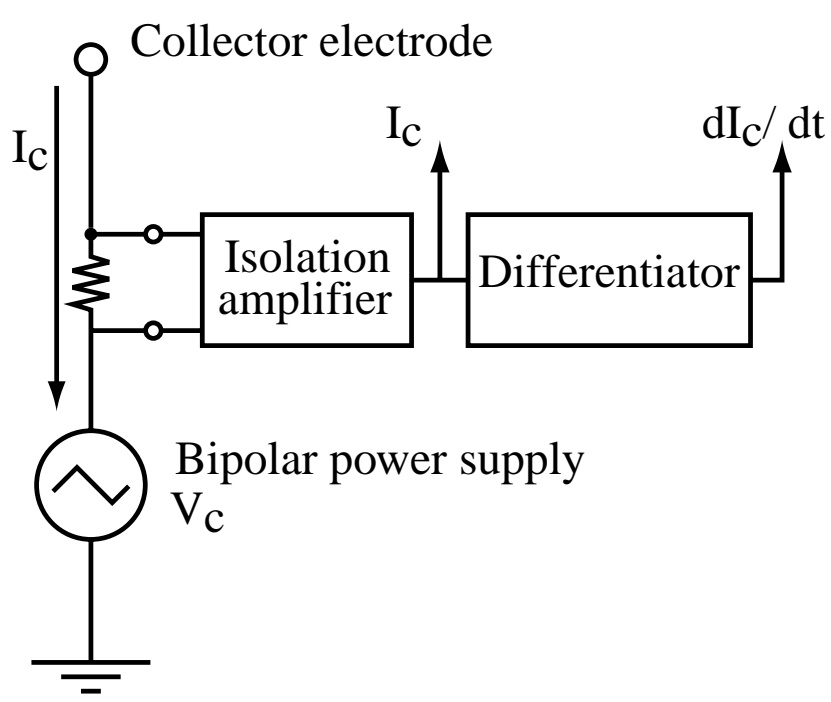

(b)

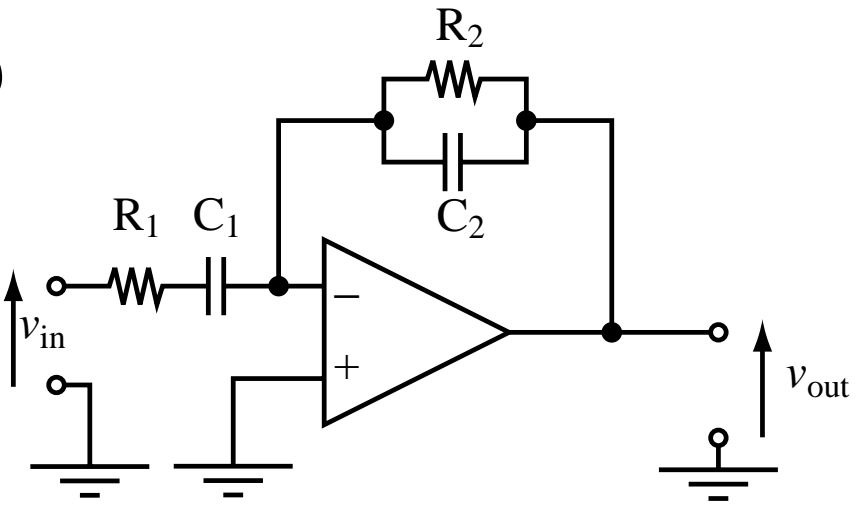

Figure 4. (a) Circuit diagram for the measurement of the IEDFs. (b) The detailed circuit diagram of the differentiator. 
(a)

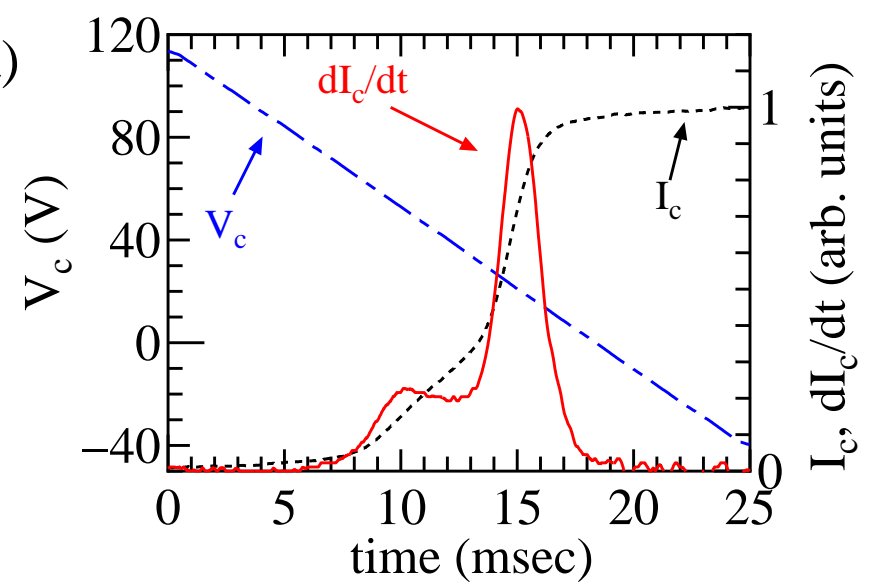

(b)

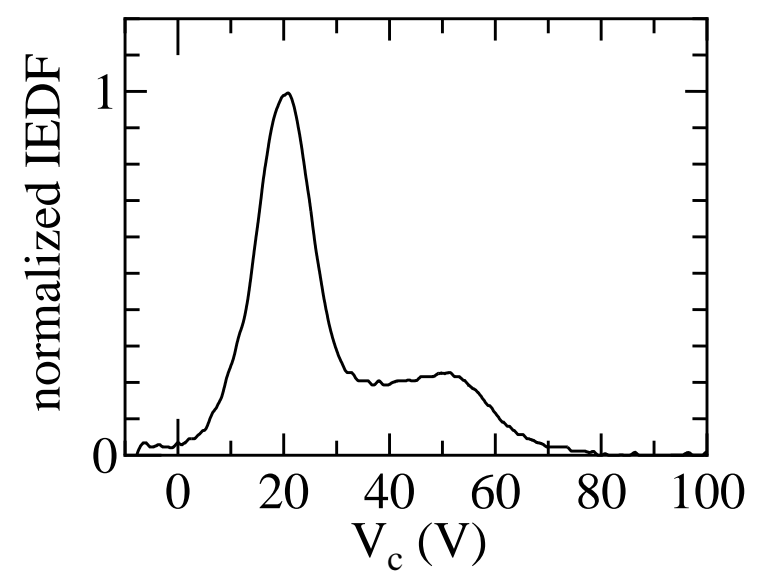

Figure 5. (a) Representative results of the collector bias voltage $V_{c}$ (dotted-dashed line), collector current $I_{c}$ (dotted line), and the first derivative $d I_{c} / d t$ (solid line) as a function of time $t$. (b) Normalized IEDF obtained from Fig. 5(a). 
(a)

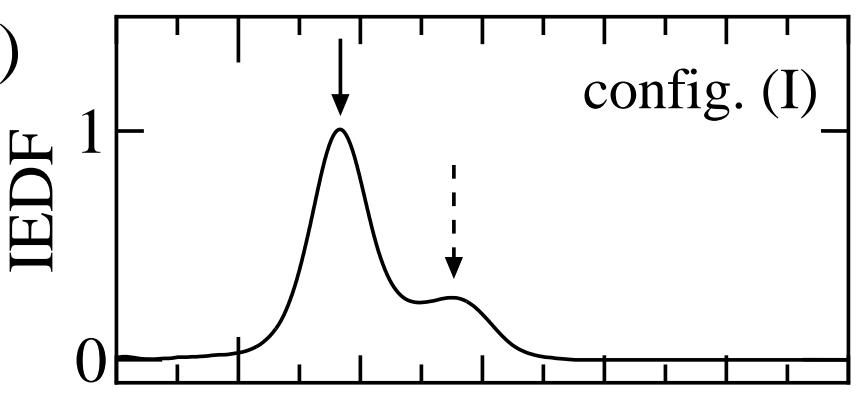

(b)

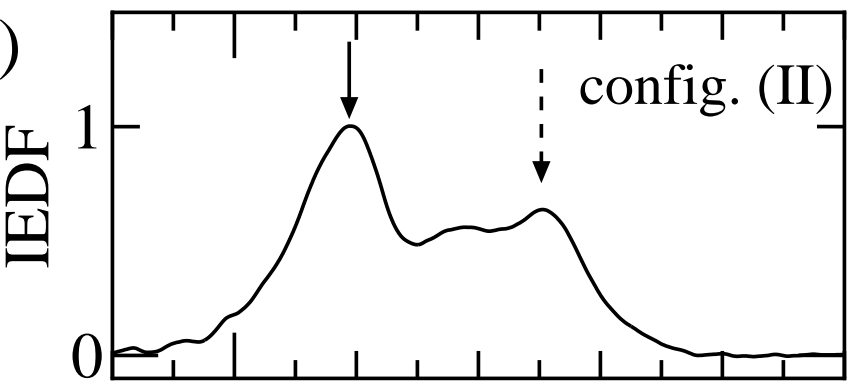

(c)

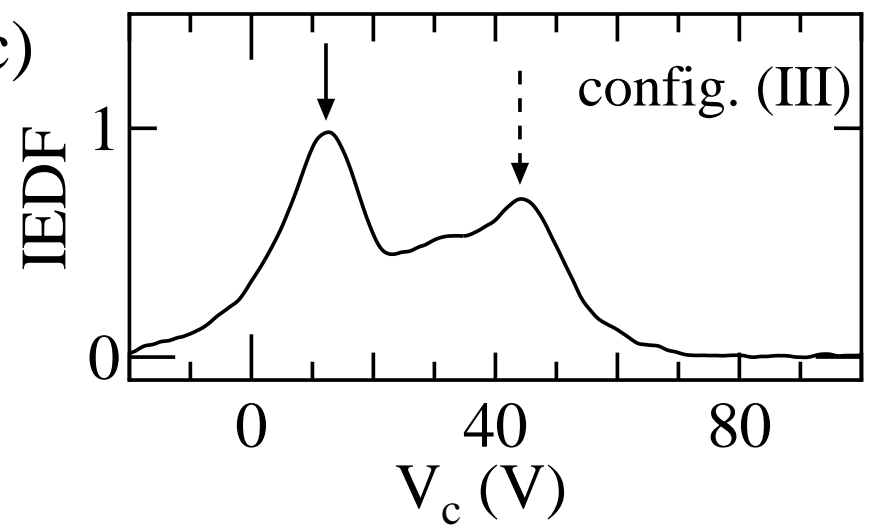

Figure 6. Normalized IEDFs at $z=3$ for configs. (I), (II), and (III). The gas pressure and the rf power are maintained at $1 \mathrm{mTorr}$ and $250 \mathrm{~W}$. The solid and dashed arrows show the local plasma potential $\phi_{p}$ and the beam potential $\phi_{b e a m}$, respectively. 
(a)

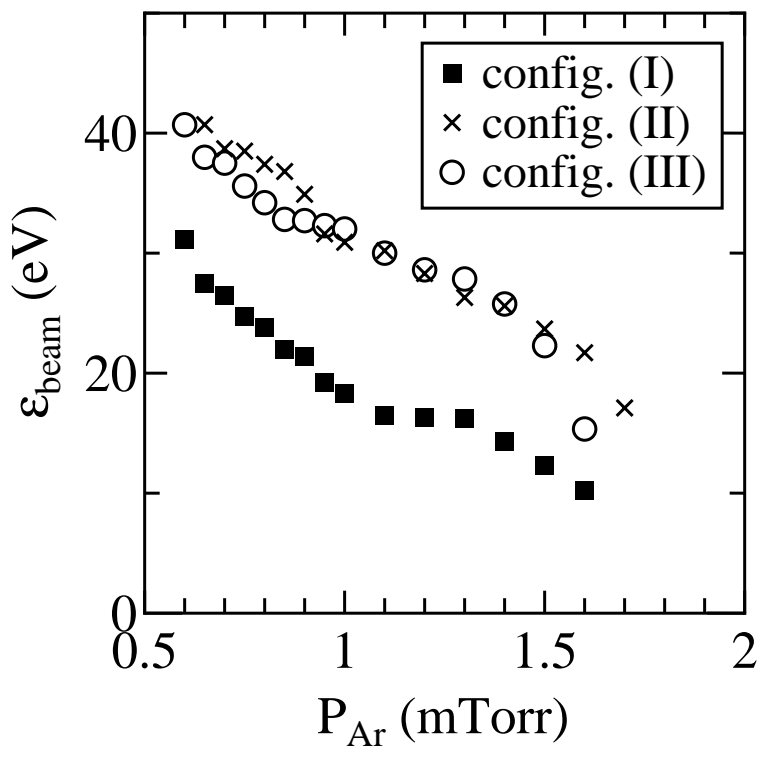

(b)

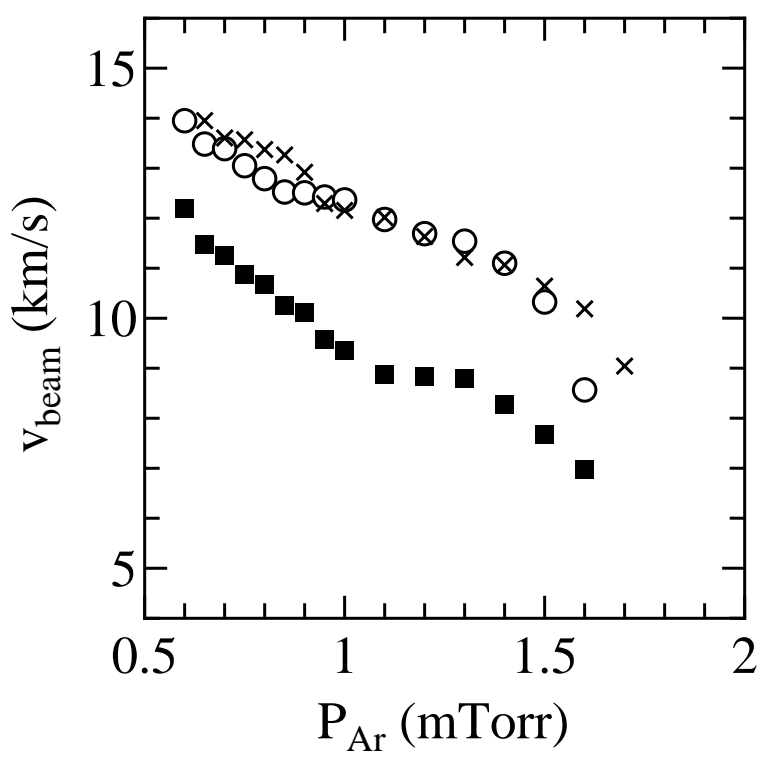

Figure 7. (a) Ion beam energies $\varepsilon_{\text {beam }}$ and (b) ion beam velocities $v_{\text {beam }}$ as a function of gas pressure $P_{A r}$ for config. (I) (closed squares), for config. (II) (crosses), and for config. (III) (open circles). 


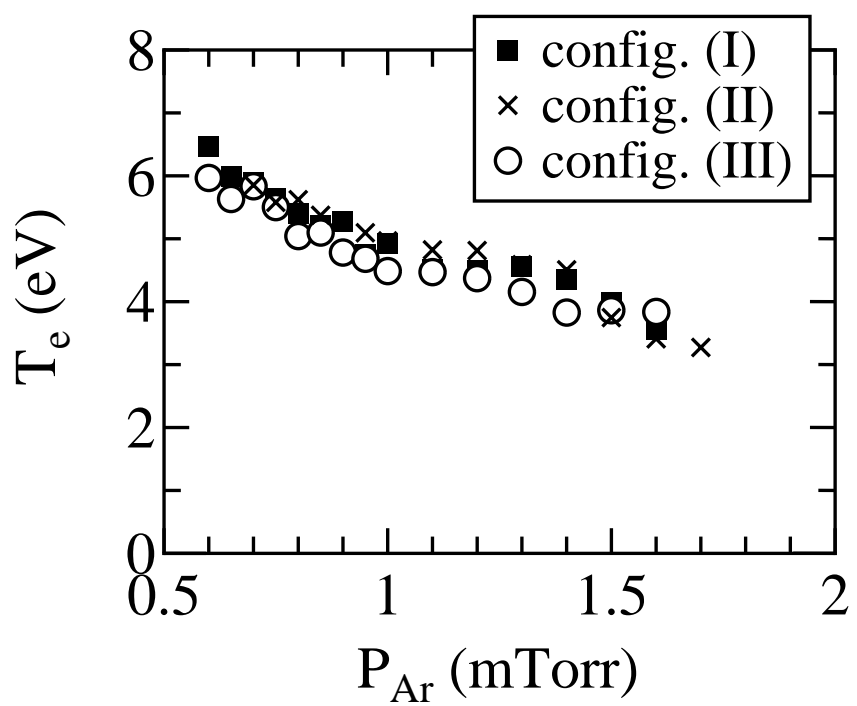

Figure 8. Electron temperatures $T_{e}$ at $z=10 \mathrm{~cm}$ as a function of gas pressure $P_{A r}$ for config. (I) (closed squares), for config. (II) (crosses), and for config. (III) (open circles). 
(a)

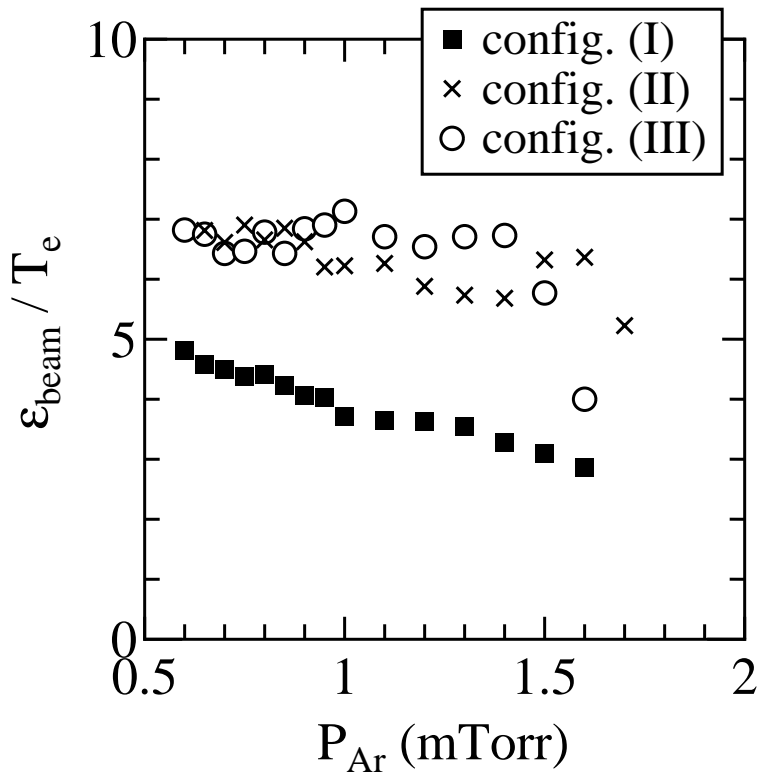

(b)

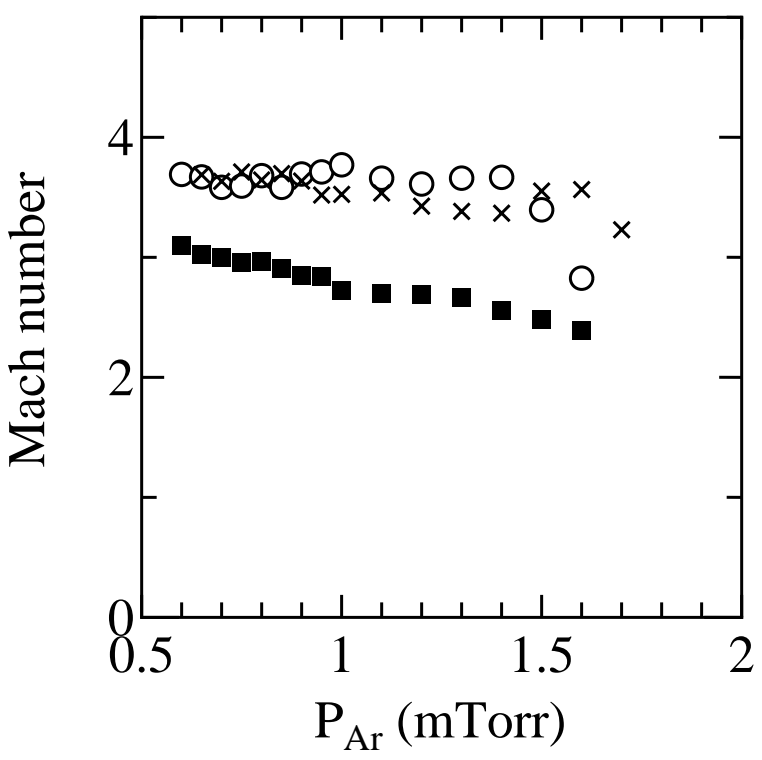

Figure 9. (a) Ion beam energies normalized by the electron temperatures $\varepsilon_{\text {beam }} / T_{e}$ and (b) mach numbers, as a function of gas pressure $P_{A r}$ for config. (I) (closed squares), for config. (II) (crosses), and for config. (III) (open circles). 